\title{
Salud mental y el rendimiento escolar en adolescentes que trabajan en los distritos de Yarinacocha y Callería - Ucayali, 2012.
}

Mental health and school performance in adolescents working in districts from Yarinacocha and CalleríaUcayali, 2012.

\section{Elsa Maximina Sánchez Yarmas ${ }^{1}$}

\section{Resumen}

El objetivo de la investigación fue de analizar la relación entre la salud mental y el rendimiento escolar en adolescentes que trabajan en los distritos de Yarinacocha y Callería - Ucayali. El diseño aplicado fue descriptivo correlacional. Se trabajó con adolescentes de 13 a 18 años pertenecientes a varias instituciones educativas de los distritos mencionados. Empleando un criterio aleatorio simple se seleccionó 204 escolares de ambos sexos (129 hombres y 75 mujeres). La variable salud mental se evaluó a través del Cuestionario de Salud Mental de Perales y cols. (1993), y la variable rendimiento escolar fue el promedio general de las calificaciones obtenidas al finalizar el año lectivo 2012. Se encontró diferencias significativas $(p<.05)$ entre la salud mental y rendimiento escolar en adolescentes de ambos sexos. Se concluyó que una baja salud mental en los adolescentes que estudian y trabajan de los distritos de Yarinacocha y Callería, se asocia a un menor rendimiento escolar.

Palabras clave: Salud mental, rendimiento escolar, adolescentes.

\section{Summary}

The aim of the research was to analyze the relationship between mental health and school performance in adolescents working in districts and Callería Yarinacocha - Ucayali. The design was descriptive correlational applied. We worked with adolescents aged 13 to 18 years belonging to various educational institutions and districts mentioned. Using a simple random criterion 204 schoolchildren of both sexes (129 males and 75 females) were selected. Mental health variable was assessed through the Mental Health Questionnaire Perales et al. (1993), and school performance variable was the overall average of the marks obtained at the end of the school year 2012. Significant differences (p $<.05$ ) between mental health and school performance in adolescents of both sexes were found. It was concluded that a low mental health in adolescents who study and work in the districts of Yarinacocha and Callería, is associated with lower school performance.

Keywords: Mental health, school performance, adolescents. 


\section{INTRODUCCIÓN}

El trabajo infantil, además de ser peligroso y perjudicial para el bienestar físico, mental o moral del niño; interfiere con su escolarización; puede exponerlo a situaciones de violencia ${ }^{(1)}$. Según las estadísticas a nivel mundial, Latinoamérica ocupa el tercer lugar de prevalencia de trabajo infantil, y el Perú es el primer país latinoamericano en promover el trabajo infantil de 5 a 11 años, con un 20\%; pero, también ocupa el tercer lugar, a nivel de Latinoamérica, en la prevalencia del trabajo infantil de 12 a 14 años, con un $10 \%{ }^{(2)}$.

Entre los efectos del trabajo infantil se encuentra la vulnerabilidad a sufrir trastornos psicosociales como conductas disociales, abuso y hostigamiento sexual y de consumo de sustancias no legales ${ }^{(3)}$.

El estudio desarrollado por el Instituto de Salud Mental ${ }^{(4)}$, reveló que más del $40 \%$ de los adolescentes de Iquitos, Tarapoto y Pucallpa identifican como problemas principales: el desempleo o la pobreza; luego sigue la violencia, la corrupción y la delincuencia. Ante esto es preciso poner especial énfasis en que la salud mental no queda circunscrita solo al sujeto que padece algún trastorno mental, sino a las condiciones de vulnerabilidad en que se encuentra, como es el caso del trabajo infantil en los adolescentes que llegan padecer a corto, mediano o largo plazo algún problema de orden conductual, cognitivo y/o emocional ${ }^{(3)}$. En tal sentido, se da la necesidad que la concepción de la salud mental debe integrar también la dimensión cultural, como parte del desarrollo personal, familiar y social, y como parte de un derecho de toda persona ${ }^{(8)}$. De otro lado, el rendimiento escolar o académico expresa el nivel de conocimientos demostrado en un área o materia comparada con la norma de edad y nivel académico ${ }^{(5)}$. Así, se tiene que el $31 \%$ de los niños y niñas de Ucayali presentan algún atraso escolar (6). Además, en la Evaluación Censal de Estudiantes (ECE) del 2011 se encontró, del total de estudiantes evaluados, que los aprendizajes esperados (nivel 2) solo fueron logrados por el $29.8 \%$ en comprensión de textos, y solo fueron logrados por el $13.2 \%$ en matemática ${ }^{(7)}$.

Este estudio se enmarca dentro de tres enfoques de intervención: Enfoque de género, donde las relaciones de género entre hombres y mujeres sean equitativas; enfoque de interculturalidad, pone de relieve la convivencia e interacción histórica entre sus culturas; $y$, el enfoque de derechos humanos al reconocer la dignidad de todos los miembros de la familia ${ }^{(8)}$.
En la literatura previa no se ha encontrado estudios que establezca la relación entre la salud mental y el rendimiento escolar en el contexto del trabajo infantil, particularmente, en la población de la región Ucayali. Los niños y adolescentes de 13 a 18 años son una población altamente demandante de espacios laborales, vulnerables a situaciones de riesgo por la actividad que desarrollan, pudiendo convertirse en un problema de salud pública que aqueja a la región de Ucayali. En consecuencia, está investigación tiene el propósito y motivación de dilucidar la relación entre las condiciones de la salud mental y el rendimiento escolar de adolescentes que trabajan en dos distritos de Ucayali.

\section{OBJETIVOS}

\section{General}

Conocer la relación entre salud mental y rendimiento escolar en adolescentes que trabajan en dos distritos de Ucayali.

\section{Específicos}

Identificar la relación entre el factor familia y apoyo social con el rendimiento escolar en adolescentes que trabajan en dos distritos de Ucayali.

Identificar la relación entre el factor uso y abuso de drogas con el rendimiento escolar en adolescentes que trabajan en dos distritos de Ucayali.

Identificar la relación entre el factor salud física y mental con el rendimiento escolar en adolescentes que trabajan en dos distritos de Ucayali.

Identificar la relación entre el factor conducta violenta con el rendimiento escolar en adolescentes que trabajan en dos distritos de Ucayali.

Identificar la relación entre el factor espacios y actividades recreacionales con el rendimiento escolar en adolescentes que trabajan en dos distritos de Ucayali.

Identificar la relación entre el factor creencias religiosas con rendimiento escolar en adolescentes que trabajan en dos distritos de Ucayali.

Identificar la relación entre el factor salud sexual y reproductiva con rendimiento escolar en adolescentes que trabajan en dos distritos de Ucayali. 


\section{HIPÓTESIS}

\section{General}

Existe relación significativa entre salud mental y rendimiento escolar en adolescentes que trabajan en dos distritos de Ucayali.

\section{Específicas}

Existe relación significativa entre el factor familia y apoyo social con el rendimiento escolar en adolescentes que trabajan en dos distritos de Ucayali.

Existe relación significativa entre el factor uso y abuso de drogas con el rendimiento escolar en adolescentes que trabajan en dos distritos de Ucayali.

Existe relación significativa entre el factor salud física y mental con el rendimiento escolar en adolescentes que trabajan en dos distritos de Ucayali.

Existe relación significativa entre el factor conducta violenta con el rendimiento escolar en adolescentes que trabajan en dos distritos de Ucayali.

Existe relación significativa entre el factor espacios $\mathrm{y}$ actividades recreacionales con el rendimiento escolar en adolescentes que trabajan en dos distritos de Ucayali.

Existe relación significativa entre el factor creencias religiosas con rendimiento escolar en adolescentes que trabajan en dos distritos de Ucayali.

Existe relación significativa entre el factor salud sexual y reproductiva con rendimiento escolar en adolescentes que trabajan en dos distritos de Ucayali.

\section{MÉTODO}

El estudio es ex post facto, retrospectivo con un grupo y correlacional ${ }^{(9)}$. La muestra quedó conformada por 204 adolescentes entre 13 y 18 años $(63.2 \%$ de varones y $36.8 \%$ de mujeres) que estudiaban en varias instituciones educativas: Colegio Nacional Yarinacocha, Hipolito Unanue, Faustino Maldonado, Teniente Diego Ferre Sosa, San Pablo Tushmo, Alfredo Vargas Guerra y Húsares del Perú). Estos estudiantes también trabajan en alguna actividad económica de los distritos de Yarinacocha y Callería. Para seleccionar la mustra se empleó un muestreo probabilístico de tipo aleatorio simple.
Para la recopilación de la información de las variables se aplicó: 1) En salud mental, el cuestionario auto administrado, constituido por preguntas del Módulo de Salud del Adolescente del cuestionario de salud mental, adaptado al Perú por Perales y cols. ${ }^{(10)}$. El rendimiento escolar fue obtenido a través del promedio general de las calificaciones al finalizar el año lectivo 2012. La información sobre el trabajo infantil fue recogido a través de cuatro preguntas: trabaja actualmente?; qué tipo de trabajo realiza?; qué tiempo tiene trabajando?; y, a qué nivel de riesgo laboral está expuesto en su trabajo?. Estas preguntas fueron incluidas dentro del cuestionario de salud mental ${ }^{(10)}$.

\section{RESULTADOS}

Los datos fueron analizados en un programa SPSS. A nivel descriptivo, se analizó cada variable y sus indicadores mediante el uso de tablas de frecuencias y porcentajes. Para fines de estudio, se realizó comparaciones según sexo. A nivel inferencial, para la verificación de la hipótesis general se utilizó el coeficiente de Chi Cuadrado, debido a que las variables eran de tipo cualitativa y con nivel de medición nominal/ ordinal.

\section{Análisis descriptivo}

\section{Caracteristicas del trabajo infantil.}

El tipo de trabajo con mayor frecuencia que presentó la muestra fue el de chofer de auto o de moto taxi, equivalente al 11.3\%; seguido de la actividad de comerciante, equivalente al $10.8 \%$; y después la labor de ambulante y ayudante de cocina, cada uno representando el 9.8\%. De acuerdo al tiempo de trabajo, se halló que el $55.4 \%$ trabajaba en promedio cuatro horas diarias; el $18.1 \%$ de la muestra presentó un promedio de de cinco horas.

\section{Rendimiento escolar.}

Se encontró un mayor rendimiento escolar en el nivel regular (varones $=71.3 \%$ y mujeres $=66.7 \%$ ). Sin embargo, también se aprecia una tendencia de nivel bajo/deficiente, en especial, para los varones (19.4\%).

\section{Salud mental.}

El nivel de salud mental, es predominantemente medio para ambos sexos (varones $=48.1 \%$ y mujeres $=38.7 \%$ ), seguido muy de cerca del nivel bajo (varones $=33.3 \%$ y mujeres $=34.7 \%$ ). 
Tabla 1. Relaciones entre la variable salud mental (y sus factores) con el rendimiento escolar ( $\mathrm{n}=204)$

\begin{tabular}{|c|c|c|}
\hline Variables relacionadas & Chi-cuadrado & gl \\
\hline Salud mental - rendimiento escolar & $106.806 * *$ & 6 \\
\hline Factor familia y apoyo social - rendimiento escolar & $65.322 * *$ & 6 \\
\hline Factor uso y abuso de drogas - rendimiento escolar & $105.399 * *$ & 6 \\
\hline Factor salud física y mental - rendimiento escolar & $84.495 * *$ & 6 \\
\hline Factor conducta violenta - rendimiento escolar & $81.884 * *$ & 6 \\
\hline Factor espacios y actividades recreacionales - rendimiento escolar & $111.913 * *$ & 6 \\
\hline Factor creencias religiosas - rendimiento escolar & $112.162 * *$ & 6 \\
\hline Factor salud sexual y reproductiva - rendimiento escolar & $105.337 * *$ & 6 \\
\hline
\end{tabular}
$* * \mathrm{p}<.01$

\section{Análisis inferencial}

Los resultados sobre las relaciones entre las variables descritas a nivel de las hipótesis se resumen en la tabla $\mathrm{N}^{\circ} 1$.

$\mathrm{Al}$ realizar el cálculo con la prueba Chi-cuadrado se encontró un $\mathrm{p}<.01$ entre las variables salud mental y rendimiento escolar; es decir, a una mayor salud mental se asocia un mejor rendimiento escolar.

De igual modo, se encontró relación significativa entre el factor familia y apoyo social con el rendimiento escolar $(\mathrm{p}<.01)$; lo cual implica, que a un mayor apoyo familiar y social se aprecia un mejor rendimiento escolar.

Lo mismo se aprecia entre el factor uso y abuso de drogas y el rendimiento escolar, se encuentran relacionadas $(\mathrm{p}<.01)$, esto es, que a mayor uso y abuso de drogas se asocia un menor rendimiento escolar.

Para la relación entre el factor salud física y mental y el rendimiento escolar se encontró un $(\mathrm{p}<.01)$, esto es, a una mayor salud física y en ausencia de síntomas psiquiátricos se espera un mejor rendimiento escolar. Se encontró que el factor conducta violenta y rendimiento escolar están asociados $(\mathrm{p}<.01)$, esto indica que ante una mayor conducta violenta se asocia un menor rendimiento académico.

Hay relación significativa entre el factor espacios y actividades recreacionales y rendimiento escolar $(\mathrm{p}<.01)$, es decir, frente a un mayor número de espacios y frecuencia de actividades recreacionales se debe esperar un mejor rendimiento escolar.

También se encontró una relación significativa entre el factor creencias religiosas y rendimiento escolar $(\mathrm{p}<.01)$, esto es, que ante una mayor creencia religiosa se espera un mejor rendimiento escolar.
Finalmente, se halló que existe relación significativa entre el factor salud sexual y reproductiva y rendimiento escolar $(p<.01)$, es decir, ante una mejor salud sexual y reproductiva se debe dar un mejor rendimiento escolar.

\section{DISCUSIÓN}

La hipótesis general fue aceptada, lo cual es respaldado por el estudio de Pedraza y Rivero ${ }^{(1)}$, al afirmar que la condición laboral afecta negativamente la asistencia escolar, aumenta sus niveles de extra edad educativa y deteriora la percepción de su estado de salud. Cabe destacar, que en Ucayali el 23,79\% de la población es adolescente. Además, el 73\% de la PEA adolescente se ubica en zonas urbanas ${ }^{(12)}$. Existe en la población de estudio un atraso escolar de alrededor del $30 \%{ }^{(13)} ; \mathrm{y}$, de acuerdo a los últimos reportes nacionales se ubica como penúltima en el tema de educación ${ }^{(14)}$. Al parecer los factores implicados en el proceso de urbanización de la población generan estresores vinculados al bajo rendimiento escolar y deterioro de la salud mental del adolescente ${ }^{(15)}$. En ese sentido, bajo las condiciones laborales no reguladas por el Estado y sin estándares internacionales claros (3), se destaca que el $32.9 \%$ de adolescentes trabaja por más de cuatro diarias y $97.5 \%$ están expuestos a algún tipo de riesgo. En estas condiciones se vulneran los derechos del niño y adolescente lo que queda reflejado en la disminución de la salud mental del adolescente que trabaja, así como en su bajo rendimiento escolar.

El rendimiento escolar está muy relacionado con el factor interacción familiar y redes de apoyo social. Este hallazgo es respaldado por la investigación de Galicia, Sánchez y Robles ${ }^{(16)}$ quienes asocian la dinámica familiar con el rendimiento escolar. En tal sentido, se ha encontrado que un entorno familiar conflictivo y con una elevada densidad habitacional en la vivienda se convierte en un factor de riesgo hacia 
la salud mental ${ }^{(10)}$. Coexisten patrones culturales y sociales que mantiene el trabajo infantil, así la familia del adolescente como trabajador es percibido como un elemento formativo ${ }^{\left({ }^{17}\right)}$. Pese a que la literatura es clara en este punto, los patrones sociales y culturales permiten la perpetuación de la condición laboral del y la adolescente.

El rendimiento escolar se relaciona con el factor de uso y abuso de sustancias (dimensión de la salud mental), lo cual pone de manifiesto que los adolescentes, en el trabajo, están expuestos al consumo de alcohol, cigarrillos y otras sustancias nocivas para el organismo. ${ }^{(17)}$. A nivel de los adolescentes la droga de mayor consumo socialmente aceptada es el alcohol (15) aunque sus consecuencias físicas y psicológicas ya han sido registradas y ampliadas por numerosos estudios ${ }^{(18)}$.

El rendimiento escolar se relaciona con el factorl de salud física y salud mental (dimensión de la salud mental), ello implica que la presencia de un estado de bienestar físico, psicológico y social se vincula con un mejor rendimiento escolar, no sólo la ausencia de alguna enfermedad o trastorno mental ${ }^{(19)}$. En este estudio los estresores psicosociales más importantes que se encontraron fueron el problemas con la familia, trabajo o estudio y el dinero; así, las emociones vinculadas a estos estresores se relacionan con la indiferencia y la desilusión. Según Page ${ }^{(20)}$, citado en Montero, Villalobos \& Valverde (2007), los factores psicosociales son facilitadores o inhibidores del rendimiento escolar, por lo cual es comprensible que una adecuada salud física y mental actúen como facilitadores del rendimiento escolar. Pese a los numerosos factores de riesgo a los que son expuestos las y los adolescentes, estos riesgos poseen un bajo nivel de demanda manifiesta.

El rendimiento escolar se relaciona con el factor de conducta violenta (dimensión de la salud mental). Al respecto se encontró que a una mayor conducta violenta se asoció un peor rendimiento escolar, existiendo una conducta violenta con predominio de nivel bajo, este hallazgo es similar al de otros estudios (21), (10). Uno de estos estudios, reveló que entre los factores de riesgo de la conducta violenta se encontraba en relación al nivel educativo de la madre, y a la percepción de mala salud y edad entre 15-17 años. Estos factores ante la situación de trabajo incrementan una mayor probabilidad de un acto violento. Asimismo, los adolescentes al encontrarse trabajando se ven expuesto a una serie de abusos y riesgos laborales que no les permiten interiorizar valores protectores ni democráticos, y llevan a repetir en sus espacios de interacción los mismos patrones autoritarios aprendidos en la relación laboral, tal como lo señala la OIT $^{(17)}$

El rendimiento escolar se relaciona con el factor de espacios y actividades recreacionales (dimensión de la salud mental). Lo cual es comprensible ante la presión y exigencia laboral y, paralelamente, la formación educativa que viene recibiendo son sopesadas por el adolescente, y dependiendo del apoyo familiar y de la percepción social le dará mayor importancia a uno de ellos ${ }^{(17),(20)}$. En este sentido, el tener espacios y participar de actividades son facilitadores de su adaptación al rol social que viene asumiendo el adolescente; más aún, cuando los adolescentes no cuentan con un parque o biblioteca en su localidad.

El rendimiento escolar se relaciona con el factor de creencias religiosas (dimensión de la salud mental), lo cual concuerda con el estudio del INSM ${ }^{(4)}$, donde la fe en Dios es un factor protector que permite aliviar los problemas de la vida que enfrenta el adolescente y la adolescente.

El rendimiento escolar se relaciona con la salud sexual y reproductiva (dimensión de la salud mental), en este aspecto entran a tallar elementos como el embarazo adolescente (el $10.7 \%$ de las mujeres que estudian y trabajan han salido embarazada, y la edad de inicio sexual es a los 12 años. Así, según la Encuesta Demográfica y de Salud Familiar - ENDES (22), el embarazo adolescente a nivel de todo el Perú fue del $13 \%$; mientras que a nivel del departamento de Ucayali la cifra se incrementa hasta el $27 \%$. Se puede deducir de estos datos que la mayor parte de las adolescentes embarazadas actualmente no se encuentran estudiando y laborando. Por lo tanto, es probable que sigan dependiendo del apoyo familiar o de sus parejas, lo cual potencialmente las excluye del acceso a actividades sociales y económicas.

\section{CONCLUSIÓN.}

Se encontró una relación directa entre salud mental y rendimiento escolar. Es decir, se asocia una baja salud mental con un menor rendimiento escolar en los adolescentes que estudian y trabajan de los distritos de Yarinacocha y Callaría. 


\section{RECOMENDACIONES}

Utilizar los datos obtenidos en la elaboración y planificación de proyectos de salud pública que brinden mejoras en la salud mental y la educación, y se les de oportunidades a los adolescentes trabajadores para que su condición social no limite sus expectativas a futuro. Es importante destacar que la salud mental en los adolescentes que trabajan está en constante vulnerabilidad, por lo cual se recomienda el desarrollo de programas de salud mental dentro de las instituciones educativas para los adolescentes, enfatizando las áreas de: uso y abuso de sustancias ilegales, comportamiento disocial, salud física y psicológica, relaciones interpersonales y salud sexual y reproductiva.

\section{REFERENCIAS BIBLIOGRÁFICAS}

1. OIT (2007). Trabajo infantil: Causa y efecto de la perpetuación de la pobreza. San José: Oficina Internacional del Trabajo.

2. OIT (2012). ¿Qué se entiende por trabajo infantil? Extraído el 12 de marzo del 2012, disponible en: http://www.ilo.org/ipec/facts/lang--es/index.htm

3. Briceño, L. \& Pinzón, A. (2004). Efectos del trabajo infantil en la salud menor trabajador. Revista de Salud Pública, 6, 270-288.

4. INMS (2005). Estudio Epidemiológico en Salud Mental en la Selva Peruana 2004. Informe General. Anales de Salud Mental, 21 ( 1 y 2).

5. Edel, R. (2003). El rendimiento académico: concepto, investigación y desarrollo. Revista Electrónica Iberoamericana sobre Calidad, Eficacia y Cambio en Educación, 1, 1-15.

6. UNICEF (2011). Estado de la Niñez en el Perú. Lima: Tarea Asociación Gráfica Educativa.

7. MINEDU (2012). Resultados de la Evaluación Censal de Estudiantes 2011 (ECE 2011). Extraído el 15 de abril del 2012, disponible en: http://umc. minedu.gob.pe/?p=230

8. Grupo de Trabajo de Salud Mental de la Coordinadora Nacional de Derechos Humanos (2006). Salud mental comunitaria en el Perú: Aportes temáticos para el trabajo con poblaciones. Lima: Ministerio de Salud, Proyecto AMARES

9. Montero, I. \& Leon, G. (2007). A guide for naming research studies in Psychology. International Journal of Clinical and Health Psychology, 7, 847-862.

10. Perales, A., Sogi, C., Sánchez, E., \& Salas, R. (1993). Adaptación del cuestionario de salud mental (para población de 12-17 años) Lima, 1993. En A. Perales \& C. Sogi (1995), Conducta violenta en adolescentes: identificación de factores de riesgo para diseño de programa preventivo (pp. 51-60). Lima: Instituto Nacional de Salud Mental "Honorio Delgado-Hideyo Noguchi".

11. Pedraza, A., \& Ribero, R. (2006). El trabajo infantil y juvenil en Colombia y algunas de sus consecuencias claves. Revista Latinoamericana de Ciencias Sociales, Niñez y Juventud, 4, 1-28.

12. MINSA (2009). Análisis de Situación de Salud de las y los Adolescentes. Ubicándolos y Ubicándonos. Lima: SINCO Editores S.A.C.

13. UNICEF (2011). Estado de la Niñez en el Perú. Lima: Tarea Asociación Gráfica Educativa.

14. Moores, P. (12 de julio de 2013). Ucayali: penúltima en educación. Gestión, pp. 17.

15. Perales, A., Sogi, C., \& Morales, R. (2001). Salud mental en adolescentes de Las Delicias (Trujillo Perú). Anales de la Facultad de Medicina, 62, 301311 .

16. Galicia, I., Sánchez, A. \& Robles, Fr. (2009). Factores asociados a la depresión en adolescentes: Rendimiento escolar y dinámica familiar. Anales de Psicología, $25,227-240$.

17. OIT (2003). III Plan Nacional para la Erradicación del Trabajo Infantil y la Protección del Trabajo Juvenil 2003-2006. Ginebra: Comité Interinstitucional para la Erradicación del Trabajo Infantil y la Protección de los Jóvenes Trabajadores.

18. Espada, J., Méndez, F., \& Hidalgo, M. (2000). Consumo de alcohol en escolares: descenso de la edad de inicio y cambios en los patrones de ingesta. Revista Adicciones, 12, 57-64.

19. OMS (2004). Invertir en Salud Mental. Ginebra: Departamento de Salud Mental y Abuso de Sustancias.

20. Montero, E., Villalobos, J., \& Valverde, A. (2007). Factores institucionales, pedagógicos, psicosociales y sociodemográficos asociados al rendimiento académico en la Universidad de Costa Rica: Un análisis multinivel. Revista Electrónica de Investigación y Evaluación Educativa, 13, 215-234

21. Cano, P., Gutiérrez, C., \& Nizama, M. (2009). Tendencia a la violencia e ideación suicida en adolescentes escolares en una ciudad de la amazonia peruana. Revista Peruana a Medicina Experimental y Salud Pública, 26, 175-181.

22. INEI (2012). Encuesta Demográfica y de Salud Familiar-ENDES. Extraído el 23 de junio del 2012, disponible en http://www.inei.gob.pe/biblioineipub/ bancopub/Est/Lib1075/index.html 